\title{
Analysis of Pharmaceuticals Inventory Management Using ABC-VEN Matrix Analysis in Selected Health Facilities of West Shewa Zone, Oromia Regional State, Ethiopia
}

\author{
Magarsa Bayissa Deressa ${ }^{1,2}$, Tamirat Bekele Beressa $\mathbb{D D}^{2}$, Awol Jemal (D) \\ 'School of Pharmacy, Institute of Health, Jimma University, Jimma, Ethiopia; ${ }^{2}$ Department of Pharmacy, College of Medicine and Health Sciences, \\ Ambo University, Ambo, Ethiopia \\ Correspondence: Tamirat Bekele Beressa, Email tamiratbekelel2@gmail.com
}

Background: The medical materials, medical supplies, and medicines cover about one-third of the annual health care organization's budget. Effective inventory management is required to balance inventory expenditure against demands for medications. The study aimed to analyze the pharmaceuticals inventory management using ABC-VEN Matrix in selected health facilities of the West Shewa zone.

Methods: A facility-based cross-sectional study was conducted in health facilities of the West Shewa zone from March 15, 2019, to May 31, 2019, and analyzed by using an MS Excel spreadsheet.

Results: At selected public health facilities of West Shewa zone in three years between September 12, 2015, and September 5, 2018, about 539 pharmaceutical items were utilized and consumed 30,837,628.61 ETB. ABC analysis indicated that 64 (11.9\%), 68 $(13.74 \%)$, and $38(11.14 \%)$ of items were classified as the class "A" items and consumed around $70 \%$ of the total pharmaceutical expenditures (TPE) at selected health facilities (aggregated), hospitals, and health centers (HC), respectively. VEN analysis revealed that 115 (35.94\%), 99 (35.61\%), and 40 (20.83\%) items were categorized as vitals and consumed 53.9\%, 51.59\%, and 36.93\% of TPE at selected health facilities. ABC-VEN matrix analysis indicated that category I items consumed $85 \%$ of TPE.

Conclusion: ABC-VEN matrix analysis of present study showed that the category I pharmaceuticals consumed a majority of TPE that need great attention for control.

Keywords: ABC analysis, VEN analysis, ABC-VEN matrix analysis, West Shewa, Oromia, Ethiopia

\section{Background}

The medical materials, medical supplies, and medicines cover about one-third of the annual health care facilities budget. ${ }^{1-3}$ To minimize the inventory expenditure, the health care facilities may keep the medicines inventory low. However, due to shortage of medicines, it is challenging to provide adequate service in the health facilities. Therefore, effective inventory management is required to balance inventory expenditure against demands medicines. ${ }^{4}$ In general, the aim of the good inventory management is to maintain a steady supply to operating units and patients while minimizing the costs of holding inventory and managing procurement. ${ }^{3}$

$\mathrm{ABC}$ analysis is one of the most effective tools the material management, based on Pareto's Law, which states $80 \%$ of the total value will be accounted $20 \%$ of the items. ${ }^{5} \mathrm{ABC}$ analysis applied for the drug consumption data over one year period or shorter. ${ }^{6}$ It classifies the pharmaceuticals into three categories. The first category is A category that have $10-20 \%$ of items which covers $70-80 \%$ of cumulative value (cost); Category B that covers $10-20 \%$ of items with the cumulative value of $15-20 \%$, and category $C$ that have $60-80 \%$ of items with the cumulative value of $5-10 \%{ }^{7}$ The A-class items need a close day to day control; the B-class items need periodic review and that of C-class items which need infrequent review. ${ }^{8}$ 
The health care facilities sometimes lack sufficient funds to buy all the desired medicines. VEN analysis is a method that helps to prioritize for purchasing medicines and keeping stock. The medicines are categorized according to their health impact, into vital, essential, and non-essential. ${ }^{6}$ Vital medicines (V) are potentially lifesaving drug and regular supply is mandatory; Essential medicines (E) are effective against less severe illness; Non-essential medicines (N) are used for minor or self-limited illness, are questionable efficacy. ${ }^{3}$

$\mathrm{ABC}$ or VEN analysis alone is sometimes not enough because of their limitations. ABC analysis mainly depends on the monetary value and misses the essentiality of the drug. Considering VEN alone, has disadvantage of non-essential drugs despite being costly, could get considered as priority drug. To overcome these limitations an ABC-VEN matrix was prepared and drugs were categorized as category I, II, and III. This effectively promotes the use of each procedure to complement each other. ${ }^{9,10}$ Category I includes all vital and expensive items (AV, BV, CV, AE, AN). Category II includes the remaining items of the $\mathrm{E}$ and $\mathrm{B}$ groups $(\mathrm{BE}, \mathrm{CE}, \mathrm{BN})$ and category $\mathrm{III}$ includes the nonessential (desirable) and cheapest group of items $(\mathrm{CN})$. Category III includes the nonessential (desirable) and a cheaper group of items $(\mathrm{CN}){ }^{7,11,12}$ The 1st Category pharmaceuticals need to be monitored and controlled continuously; the 2nd Category need to be controlled periodically, and 3rd Category does not need to be controlled periodically. ${ }^{13}$

Majority of the leading cause of disease and disability in developing countries can be prevented, treated or alleviate with cost effective essential medicines. ${ }^{3}$ However, The World Health Organization (WHO) reported that about $30 \%$ of the world population lacks regular access of essential medicines; ${ }^{14,15}$ and in Africa and Asia it rises to over $50 \%{ }^{14}$ Different studies conducted in Ethiopia and other African countries indicated that unavailability of essential pharmaceuticals in health facilities is challenging issues. A study conducted in eight sub-Saharan African countries indicated that the availability of 12 priority essential medicines for women is ranged from $22 \%$ to $40 \%$ and for children it ranged from $28 \%$ to $57 \% .{ }^{16}$ Another study conducted on ten tracer drugs in 15 health centers (HC) of Rwanda showed that $73 \%$ of the facilities faced a challenge of medium to high levels of stock outs. ${ }^{17}$ The study conducted at Adama city (Ethiopia) HCs indicated that the average availability of eleven tracer drugs was $76.3 \% .{ }^{18}$ Another study conducted at South West Shewa zone (Ethiopia) showed that wastage of medicines in the facilities is increasing from time to time. ${ }^{19}$

There has been no previous study conducted concerning pharmaceutical inventory management in the West Shewa zone and the status of pharmaceutical inventory management concerning budget spent on vital, essential, and nonessential pharmaceuticals was unknown. This study aimed to assess inventory management by ABC-VEN matrix analysis of pharmaceuticals in health facilities of West Shewa zone.

\section{Methods}

\section{Study Area and Study Period}

The study was conducted in public health facilities of the West Shewa zone. West Shewa zone has 22 districts and the total population was 2,058,676 according to 2007 census data. ${ }^{20}$ Out of 581 public health facilities giving service in the zone; 8 of them were hospitals, $91 \mathrm{HCs}$, and 482 health posts. A total of 3513 health professionals in the zone (1541 Males and 1972 Females) and from this, 84 of them were Pharmacists (68 Male and 16 Female) and 102 were druggists (79 Male and 23 Female). The study was conducted between March 15, 2019, and May 31, 2019.

\section{Study Design}

A health facility based cross-sectional study was conducted in which ABC-VEN matrix analysis was used to analyze pharmaceutical inventory management practice of health facilities of West Shewa zone.

\section{Population}

Source Population

The source population of the study was all health facilities like HCs, hospitals, and health posts found in the West Shewa zone, all pharmaceutical products used in this zone, and records used in inventory managements like health commodity management information system (HCMIS), bin cards, stock cards, goods receiving voucher (model no 19) and goods issuing voucher (model no 22). 


\section{Study Population}

The study population includes all budget pharmaceuticals which were collected from 15 selected public health facilities' pharmaceutical records.

\section{Inclusion and Exclusion Criteria Inclusion Criteria}

Both hospitals and HCs included in the study were public health facilities. Pharmaceuticals included in the study were budget drugs, laboratory reagents and chemicals, and medical supplies.

\section{Exclusion Criteria}

Hospitals and HCs who did not start giving service before September 2008 EC (September 2015 GC) were not included in the study. Health posts were excluded from the study because they are under the umbrella of HCs and most logistic activities are conducted at $\mathrm{HC}$.

\section{Sample Size Determination and Sampling Techniques}

\section{Sample Size Determination}

The total number of public hospitals and HCs in this zone was 99 (91 HCs plus 8 hospitals). The number of health facilities required for the study was calculated based on the recommendation of the USAID delivery project logistics indicators assessment tool (LIAT) which states a minimum of $15 \%$ of the available health facilities needs to be considered. ${ }^{21}$

The number of health facilities required for this study was calculated by (total number of hospitals and HCs X $15 \%=$ $99 \times 0.15=14.85$ ). A total of 15 health facilities were needed for the study. The number of hospitals and HCs required for the study was calculated proportionally. The number of hospitals required was ((Total number of hospitals/total number of hospitals and HCs) X sample size $=(8 / 99) \mathrm{X} 15=1.21)$ was 2 and that of $\mathrm{HC}$ was $13(15$ minus 2$)$.

\section{Sampling Techniques}

To take samples to be included in the study health facilities were categorized into hospitals and HCs. The hospitals were then categorized into referral hospitals (but it was excluded since it started working at the end of 2008 EC), general hospitals, and district hospitals. From 4 district hospitals available in the zone; three of them were excluded by their service year and the left 1 hospital was included in the study. From three available general hospitals, one of them was selected by simple random sampling using the lottery method. To select HCs to be included for the study, first from a total of 22 districts found in the zone, 13 of them were selected by simple random sampling method, and then from each of the selected districts, one $\mathrm{HC}$ was selected using the lottery method.

\section{Study Variables \\ Dependent Variables}

The dependent variables were the proportion of pharmaceuticals categorized as class A, B, and C as per ABC Analysis and their expenditures, the proportion of pharmaceuticals categorized as class V, E, and N as per VEN analysis, and their expenditures.

\section{Independent Variables}

Independent variables of the study were pharmaceuticals consumed, purchasing cost of pharmaceuticals, and prevalence and severity of the disease.

\section{Data Collection Procedures}

Data used for $\mathrm{ABC}$ analysis was the quantity of annual consumption and unit price of each pharmaceutical used in the health facilities. The data of three years' consumption between September 12, 2015, and September 5, 2018, was collected by reviewing issue documents (model no. 22 and HCMIS (if the facility was using it). 
The tool used for collecting data for VEN analysis was taken from Management science for health. ${ }^{3}$ Tool used for data collection of VEN analysis of medical supplies is not the same as that of medicine and was developed from Management Science for $\mathrm{Health}^{3}$ by considering their criticality in giving basic health care service and their supply behavior.

\section{Data Processing and Analysis}

\section{The Procedure of $A B C$ Analysis}

The annual expenditure of individual items was calculated and arranged in descending order. The cumulative cost of all the items was calculated. Percentage expenditure of individual pharmaceuticals was calculated and then the cumulative percentage of items and the cumulative percentage the expenditure were calculated. Then, the list of the items was divided into three categories: A, B, C, based on the percentage of the cumulative cost of $70 \%, 20 \%$, and $10 \%$ respectively. ${ }^{10,22}$ The VEN categories were carried out according to the WHO guideline. ${ }^{3}$

The ABC-VEN matrix was conducted by cross-tabulating the ABC and VEN analysis. From the combination of cross tabulation three categories were obtained. Category I consists of items belonged to AV, AE, AN, BV, and CV subcategories; category II had items in $\mathrm{BE}, \mathrm{CE}, \mathrm{BN}$, and Category III contained the remaining items which is $\mathrm{CN} .{ }^{10}$

\section{Results}

This study was conducted in fifteen public health facilities of West Shewa zone. Thirteen of them were HCs and two of them were hospitals. The finding of the study was presented in three different categories namely: aggregated result of all 15 selected health facilities, aggregated result of two selected hospitals and aggregated result of 13 selected HCs. In each category, $\mathrm{ABC}$ analysis, VEN analysis, ABC-VEN matrix analysis was presented separately.

\section{ABC Analysis}

\section{ABC Analysis of Pharmaceuticals of Selected Health Facilities of West Shewa Zone}

At health facilities selected from West Shewa zone in three years between September 12, 2015, and September 5, 2018, about 539 pharmaceutical items were utilized and consumed 30,837,628.61 ETB. ABC analysis showed that 64 (11.9\%) items were categorized as class A and consumed around $70.34 \%$ of total pharmaceutical expenditures (TPE). Class B and $\mathrm{C}$ accounted for 97 (18\%) and 378 (70.1\%) items that consumed 19.68\% and 9.98\% of TPE respectively (Table 1).

Out of 64 items found in class A amoxicillin $500 \mathrm{mg}$ capsule, surgical glove latex (sterile) number 7.5 and ceftriaxone $1 \mathrm{gm}$ injection contributed $15.1 \%$ of TPE (Figure 1).

$\mathrm{ABC}$ analysis of the hospitals, HCs, and aggregated data are compatible under V. Pareto curve. From the graph relatively $\mathrm{ABC}$ analysis curve of $\mathrm{HCs}$ at class $\mathrm{A}$ is steeper than others and shows that pharmaceuticals of this class took the maximum percentage of budget range than others. On the other hand, at hospitals, the $\mathrm{ABC}$ analysis curve of class $\mathrm{A}$ is less steep than the other two and then it shows as pharmaceuticals in this class took a lower portion of the budget range and maximum of items proportion (Figure 2).

Table I Summary of ABC Analysis of Pharmaceuticals of Selected Health Facilities of West Shewa Zone, Oromia, Ethiopia, January Selected Health Facilities of West Shewa Zone, Oromia, Ethiopia, January 2020

\begin{tabular}{|l|l|l|l|}
\hline Class & Number of Items (\%) & Consumption Value in ETB & Value in \% \\
\hline A & $64(11.9 \%)$ & $21,690,111.68$ & $70.34 \%$ \\
\hline B & $97(18 \%)$ & $6,069,077.62$ & $19.68 \%$ \\
\hline C & $378(70.1 \%)$ & $3,078,439.31$ & $9.98 \%$ \\
\hline Total & $539(100 \%)$ & $30,837,628.61$ & $100 \%$ \\
\hline
\end{tabular}




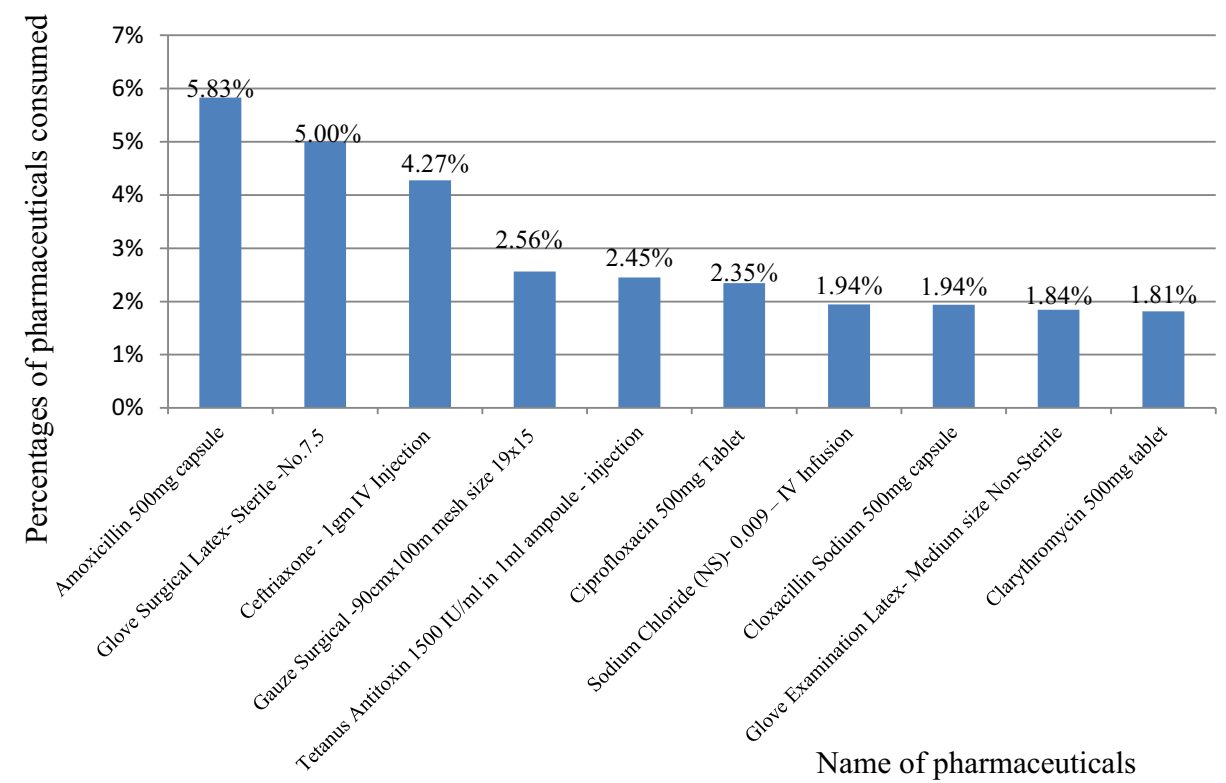

Figure I Top ten most costly pharmaceuticals of selected health facilities of West Shewa zone, Oromia, Ethiopia, January 2020.

$\mathrm{ABC}$ Analysis of hospitals, HCs and aggregated data

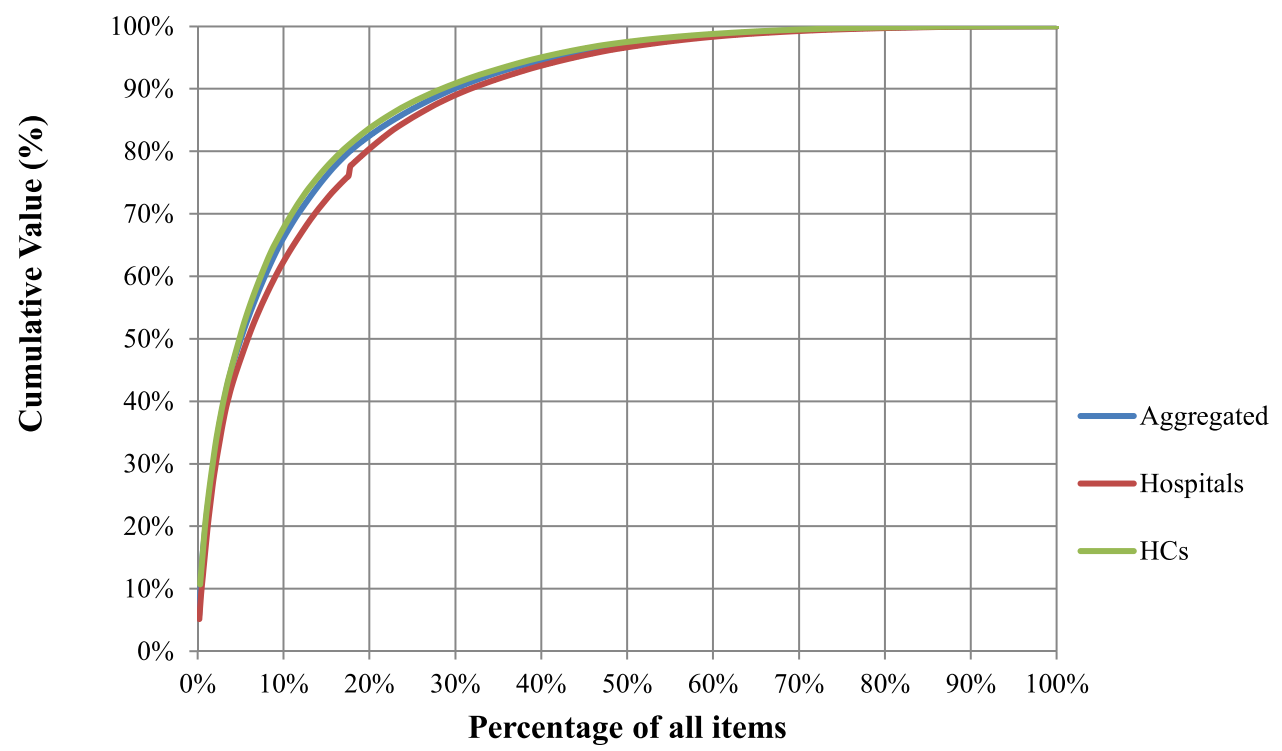

Figure 2 Pharmaceuticals' ABC Analysis curve of Hospitals, HCs and aggregated data of West Shewa zone, Oromia, Ethiopia, January 2020.

\section{ABC Analysis of Selected Hospitals of West Shewa Zone}

In the three years at selected hospitals of the West Shewa zone, around 495 items were utilized and consumed 19,241,061.7 ETB. ABC analysis of these items showed that 68 (13.74\%) items were accounted for class A and consumed around $70.18 \%$ of TPE (Table 2).

Out of 68 of class A surgical glove number 7.5 and ceftriaxone $1 \mathrm{gm}$ injection contributed around $9.1 \%$ of total APE (Figure 3). 
Table 2 Summary of ABC Analysis of Selected Hospitals of West Shewa Zone, Oromia, Ethiopia, January 2020

\begin{tabular}{|l|l|l|l|}
\hline Class & Number of Items (\%) & Consumption Value in ETB & Value (\%) \\
\hline A & $68(13.74 \%)$ & $13,502,614.5$ & $70.18 \%$ \\
\hline B & $90(18.18 \%)$ & $3,831,682.63$ & $19.91 \%$ \\
\hline C & $337(68.08 \%)$ & $1,906,764.57$ & $9.91 \%$ \\
\hline Total & $495(100 \%)$ & $19,241,061.7$ & $100.00 \%$ \\
\hline
\end{tabular}

\section{ABC Analysis of Selected Health Centers of West Shewa Zone}

At selected HCs of West Shewa zone around 341 pharmaceuticals were utilized and consumed 11,635,306.17 ETB. ABC analysis showed that 38 (11.14\%) number of items were categorized as class A and consumed $70.40 \%$ of TPE (Table 3 ).

At selected HCs out of 38 items classified as class A only amoxicillin $500 \mathrm{mg}$ capsule consumed $10.7 \%$ total TPE (Figure 4).

\section{VEN Analysis}

VEN Analysis of Pharmaceuticals of Selected Health Facilities of West Shewa Zone

At the selected health facilities of the West Shewa zone, a total of a total of 320 Pharmaceuticals were identified VEN analysis. From this 115 (35.94\%) of them were categorized as vitals (Table 4).

\section{VEN Analysis of Selected Hospitals at West Shewa Zone}

VEN analysis of selected hospitals at West Shewa zone revealed that a total of 278 pharmaceuticals were identified. From this 99 (35.61\%), 138 (49.64\%) and 41 (14.75\%) number of pharmaceuticals were classified as vitals, essentials and nonessential respectively. Vital items consumed 7,380,201.70 ETB (51.59\%) of TPE (Table 5).

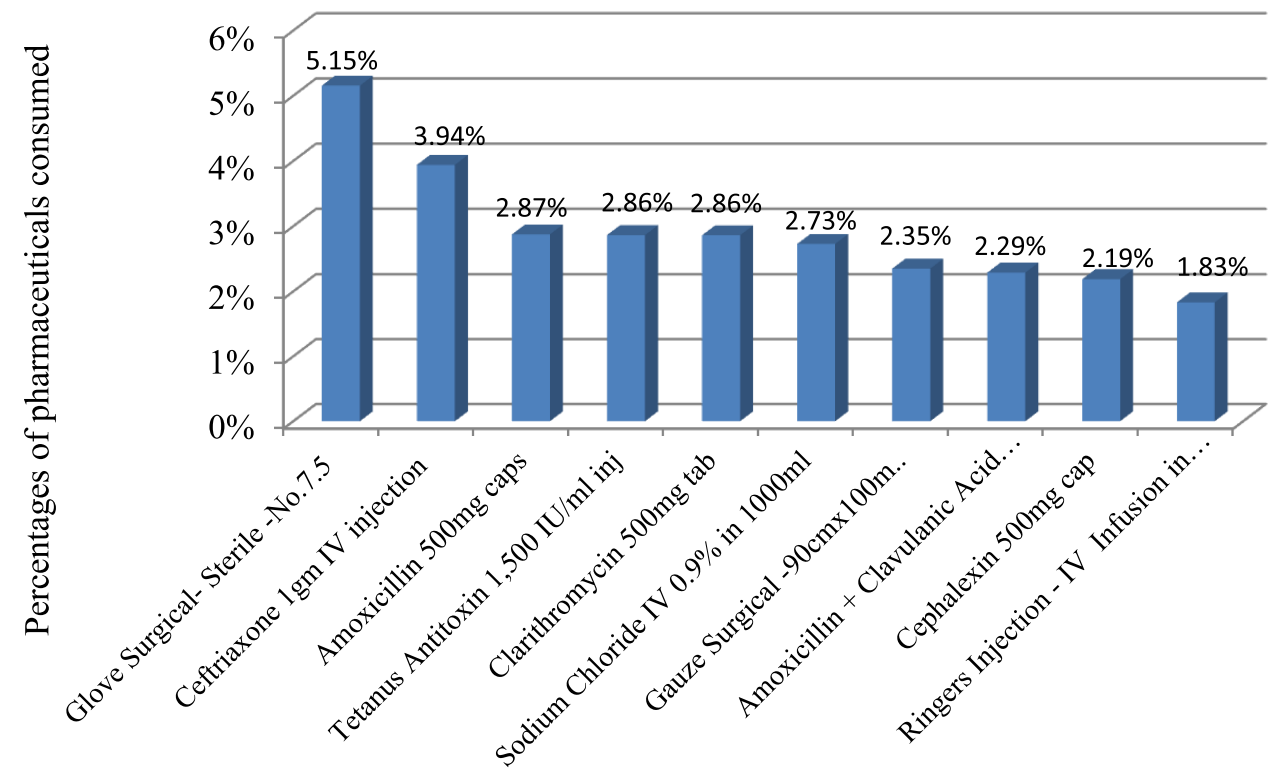

Name of pharmaceuticals

Figure 3 Top ten costly items of selected hospitals at West Shewa zone, Oromia, Ethiopia, January 2020. 
Table 3 Summary of ABC Analysis of Selected HCs of West Shewa Zone, Oromia, Ethiopia, January 2020

\begin{tabular}{|l|l|l|l|}
\hline Class & Number of Items (\%) & Consumption Value in ETB & Value (\%) \\
\hline A & $38(11.14 \%)$ & $8,191,559.04$ & $70.40 \%$ \\
\hline B & $61(17.89 \%)$ & $2,321,154.46$ & $19.95 \%$ \\
\hline C & $242(70.97 \%)$ & $1,122,592.67$ & $9.65 \%$ \\
\hline Total & $341(100.00 \%)$ & $11,635,306.17$ & $100.00 \%$ \\
\hline
\end{tabular}

VEN Analysis of Pharmaceuticals at Selected Health Centers of West Shewa Zone

A total of 192 pharmaceuticals were identified and classified by VEN Analysis in selected HCs of West Shewa zone. From this 40 (20.83\%), $116(60.42 \%)$ and $36(18.75 \%)$ of items were categorized under vital, essential and non-essential class respectively (Table 6).

\section{ABC-VEN Matrix Analysis}

ABC-VEN Matrix Analysis Pharmaceuticals of Selected Health Facilities at West Shewa Zone

The finding of ABC-VEN matrix analysis at selected health facilities of West Shewa zone revealed that $142(44.4 \%)$ of items classified as category I pharmaceuticals and consumed $85.5 \%$ of TPE. 137 (42.8\%) and 41 (12.8\%) of items classified as category II and III consumed $13.3 \%$ and $1.3 \%$ TPE respectively (Figure 5).

\section{ABC-VEN Matrix Analysis of Pharmaceuticals at Selected Hospitals of West Shewa Zone}

ABC-VEN matrix analysis of pharmaceuticals at selected hospitals of West Shewa zone revealed that 123 (44.24\%), 120 $(43.17 \%)$ and $35(12.59 \%)$ number of items contribute category I, II, and III that consumed $85.25 \%, 13.37 \%$, and $1.38 \%$ of TPE respectively.

\section{ABC-VEN Matrix Analysis of Pharmaceuticals at Selected Health Center of West Shewa Zone}

The ABC-VEN matrix analysis of pharmaceuticals at selected HCs of the West Shewa zone revealed that 61 (31.77\%) items were category I and consumed $82.33 \%$ of TPE. $105(54.69 \%)$ and $26(13.54 \%)$ items contribute to category II and III that consumed $16.55 \%$ and $1.13 \%$ of TPE (Table 7).

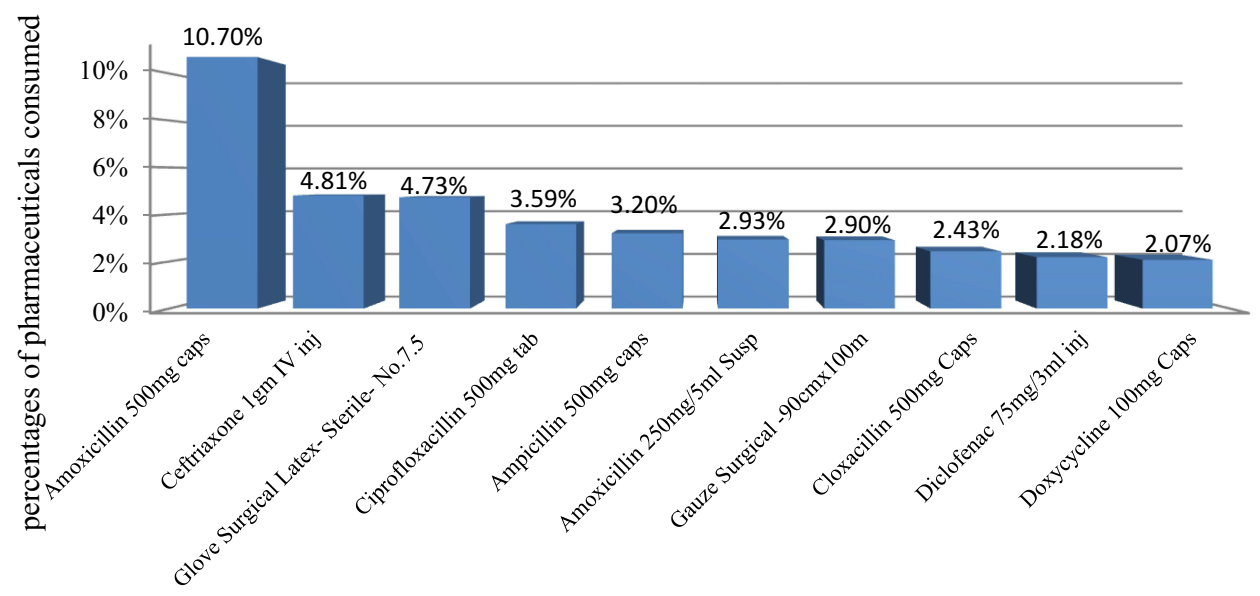

Name of pharmaceuticals

Figure 4 Top ten costly items of selected HCs at West Shewa zone, Oromia, Ethiopia, January 2020. 
Table 4 VEN Analysis of Pharmaceuticals of Selected Health Facilities of West Shewa Zone, Oromia, Ethiopia, January 2020

\begin{tabular}{|l|l|l|l|}
\hline VEN Analysis & Number of Items (\%) & Consumption Value (in ETB) & Value (\%) \\
\hline V & $115(35.94 \%)$ & $14,243,893.81$ & $53.9 \%$ \\
\hline E & $156(48.75 \%)$ & $1 \mathrm{I}, 162,220.06$ & $42.2 \%$ \\
\hline N & $49(15.31 \%)$ & $1,032,168.31$ & $3.9 \%$ \\
\hline Total & $320(100 \%)$ & $26,438,282.18$ & $100 \%$ \\
\hline
\end{tabular}

Table 5 Summary of VEN Analysis of Selected Hospitals of West Shewa Zone, Oromia, Ethiopia, January 2020

\begin{tabular}{|l|l|l|l|}
\hline VEN Category & Number of Items (\%) & Consumption Value (in ETB) & Value (\%) \\
\hline V & $99(35.61 \%)$ & $7,380,201.70$ & $51.59 \%$ \\
\hline E & $138(49.64 \%)$ & $6,423,565.65$ & $44.90 \%$ \\
\hline N & $41(14.75 \%)$ & $501,744.27$ & $3.51 \%$ \\
\hline Total & $278(100.00 \%)$ & $14,305,511.62$ & $100.00 \%$ \\
\hline
\end{tabular}

Table 6 Summary for VEN Analysis of Pharmaceuticals of Selected HCs of West Shewa Zone, Oromia, Ethiopia, January 2020

\begin{tabular}{|l|l|l|l|}
\hline VEN Analysis & Number of Items (\%) & Consumption Value (in ETB) & Value (\%) \\
\hline V & $40(20.83 \%)$ & $3,761,507.51$ & $36.93 \%$ \\
\hline E & $116(60.42 \%)$ & $5,728,539.28$ & $56.24 \%$ \\
\hline N & $36(18.75 \%)$ & $696,596.98$ & $6.84 \%$ \\
\hline Total & $192(100.00 \%)$ & $10,186,643.77$ & $100.00 \%$ \\
\hline
\end{tabular}

\section{Discussion}

The present study assessed the pharmaceutical inventory management of health facilities of west Shewa zone by using ABC-VEN Matrix Analysis. Conducting ABC analysis or VEN analysis alone has its limitations. ${ }^{7} \mathrm{ABC}$ analysis has a limitation of ignoring the criticality of pharmaceuticals and VEN analysis alone also ignore the cost value of pharmaceuticals. ${ }^{7}$ Therefore, to use complementary advantages of these methods ABC-VEN matrix analysis is needed to identify pharmaceuticals that need stringent control.

$\mathrm{ABC}$ analysis of the present study was comparable with the previous study conducted by Ceylan and Bulkan ${ }^{23}$ in which $10.31 \%$ class A items consumed $69.96 \%, 21.78 \%$ class B items consumed $20.02 \%$, and $67.9 \%$ class C items consumed $10.01 \%$ of pharmaceutical expenditures. It was also comparable with the study conducted at a secondary level hospital in India in which $10.4 \%$ class A, $19.8 \%$ class B, and $69.8 \%$ class C items consumed $69.7 \%, 21.2 \%$, and $9.10 \%$ of pharmaceutical expenditures respectively. ${ }^{22}$ It was also comparable with a similar study conducted at Tertiary Care Teaching, Research and Referral Healthcare Institute of India by Nigah et al ${ }^{24}$ in which $13.78 \%$ (class A items), $21.85 \%$ (class B) and $64.37 \%$ (class C) consumed $69.97 \%, 19.95 \%$ and $10.08 \%$ of pharmaceutical expenditures respectively. Similarity of these studies with the present study might be related with that classification of ABC analysis follows V. Pareto classification way and in ranges he recommended. 


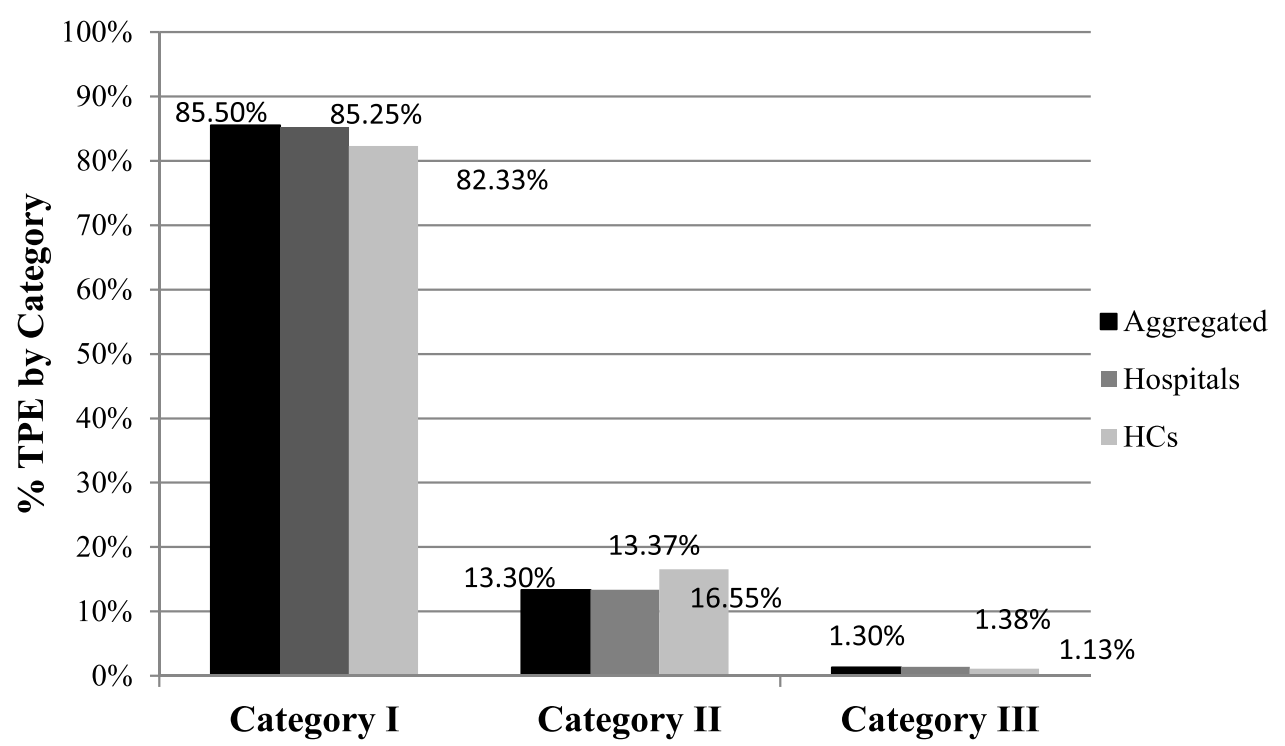

Figure 5 ABC-VEN matrix analysis of pharmaceuticals consumption at different level of health facilities of West Shewa zone, Oromia, Ethiopia, January 2020. Category I items belonging to $\mathrm{AV}, \mathrm{AE}, \mathrm{AN}, \mathrm{BV}$, and $\mathrm{CV}$ subcategories; category II: $\mathrm{BE}, \mathrm{CE}$, and $\mathrm{BN}$ subcategories; and category III: items in the $\mathrm{CN}$ subcategory.

However, the present study was different from a study conducted at the hospital in Turkey by. GünerGören and Dağdeviren ${ }^{25}$ where only $10 \%$ of class A items consumed $80 \%$ of pharmaceutical expenditures, $23 \%$ (class B items) and $67 \%$ (class $\mathrm{C}$ items) consumed $17 \%$ and $3 \%$ of pharmaceutical expenditures respectively. The finding of the present study had a big gap with a study conducted at Black Lion specialized hospital (Ethiopia) in which $2.99 \%$ (for 2008), $0.25 \%$ (for 2009), and $1.48 \%$ (for 2010) items were assigned as class $A$ and consumed $79 \%, 77 \%$ and $80 \%$ of the total medicines budget respectively. The Class B items in each year were $14.54 \%, 11.4 \%$, and $17.8 \%$ accounting for $16.21 \%, 17.79 \%$, and $16.19 \%$ of the medicines budget respectively. Class $\mathrm{C}$ medicines representing $82 \%, 88 \%$, and $81 \%$ of the items consumed $5.1 \%, 4.93 \%$, and $4.94 \%$ of the budget in each year respectively. ${ }^{26}$ This difference might be due to the difference of level of health facility, pharmaceutical products included for the study and difference of pharmaceutical budget allocated.

Of the top ten expensive pharmaceuticals of class A at selected hospitals, five of them were antibiotics and at HCs it was increased to seven. Amoxicillin $500 \mathrm{mg}$ capsule and ceftriaxone $1 \mathrm{gm}$ injection were commonly available at both levels of health facilities. The similarity and more consumption of these antibiotics might be due to the high prevalence of infectious disease in the study area or the prescribers might prescribe antibiotics for every suspected case without confirmation of microbes.

At selected health facilities of the zone and hospitals around one-third of pharmaceuticals identified by VEN analysis were vitals that consumed more than half of the pharmaceutical budget. Since these items are life-saving their stock out can harm health care service provision and they need continuous inventory control. They should be available always and it is mandatory to supply them regularly. If there is a lack of budget to purchase all needed pharmaceuticals, they should

Table 7 Summary of Category of ABC-VEN Matrix Analysis of Pharmaceuticals of Selected HCs of West Shewa Zone, Oromia, Ethiopia, January 2020

\begin{tabular}{|l|l|l|l|}
\hline Category & Number of Items (\%) & Consumption Value (ETB) & Value (\%) \\
\hline I (AV+BV+CV+AE+AN) & $61(31.77 \%)$ & $8,385,646$ & $82.33 \%$ \\
\hline II (BE+CE+BN) & $105(54.69 \%)$ & $1,685,690$ & $16.55 \%$ \\
\hline III (CN) & $26(13.54 \%)$ & $115,307.17$ & $1.13 \%$ \\
\hline Total & $192(100.00 \%)$ & $10,186,643.17$ & $100.00 \%$ \\
\hline
\end{tabular}


get priority and purchase. Around half of pharmaceuticals listed by VEN analysis of this study were essentials and they need medium inventory control. Few items of the present study were non-essentials and their stock out does not affect giving health care service of health facilities. Then least priority would be given to these items while purchasing pharmaceuticals. Attention for control of pharmaceuticals while their selection, procurement, keeping their stock and use should be given first for vital items then for essentials and lastly non-essential items should be considered.

Considering VEN analysis alone of the present study of the selected health facilities of West Shewa zone can effectively control 115 vital items with 54\% of TPE and 156 essential items with $42.3 \%$ of TPE which gives a total of 96.3\% of TPE, but it would compromise expensive (items which consume high pharmaceutical budget) items of class A which found in non-essential category.

The finding of the VEN analysis of the present study was different from the study conducted by Ceylan and Bulkan ${ }^{23}$ in which $10.67 \%$ vital items (consumed $23.31 \%$ of pharmaceutical budget), $45.49 \%$ essential items (consumed $55.85 \%$ ), and $43.75 \%$ non-essential items (consumed 20.84\%). This study was also different from the study conducted at Tertiary care Teaching, Research and Referral Health care Institute of India by Nigah et $\mathrm{al}^{24}$ and a study conducted in Sudan by Mousnad et al. ${ }^{27}$ The finding of the study conducted in India was $12.11 \%$ vital, $59.38 \%$ essential and $28.51 \%$ nonessential items that consumed $17.14 \%, 72.38 \%$, and $10.48 \%$ respectively. ${ }^{24}$ The findings of the study conducted in Sudan were $2.34 \%$ vital items (consumed $5.46 \%$ of pharmaceutical expenditure), $52.65 \%$ essential (consumed $68.11 \%$ ), and $45.01 \%$ non-essential items (consumed 26.43\%). ${ }^{27}$ Both in terms of percentages of items and TPE vital items of the present study was higher than previously conducted studies and that of non-essential items of the present study were smaller than these previous studies. This variation might be due to the aggregation of findings of different health facilities by this study. Each level of health facility serves different health care conditions and they may use different items for different health conditions. Additionally, a drug that is vital at one health facility may be essential or non-essential (completely not needed) at others, and it may vary the consumption value of pharmaceuticals from other studies.

ABC-VEN matrix analysis of the present study showed that category I items consumed a high percentage of TPE and it enhances the ability to narrow down attention on these items for strict managerial control since these items are costly or expensive. Category II items consumed a medium percentage of TPE and the majority of them were essential items (BE, $\mathrm{CE})$ and they need moderate inventory control by middle-level managers. ${ }^{28}$

Category I items of ABC-VEN matrix analysis need continuous monitoring and control. At health facilities of West Shewa zone 142 items belonged to category I (consumed 85.5\% of TPE) only AV, AE, and BV subgroups of this category consists of $81(25.31 \%)$ items which consumed $82.1 \%$ of TPE and the stock out of these items can affect the service given since they are either vital or essential. To manage the capital insufficiency due to these items, low buffer stock needs to be maintained while keeping strict control on the consumption level and stock on hand. ${ }^{28}$ From category I, 59 (18.44\%) pharmaceuticals belonged to CV and they consumed 2.3\% of TPE, these items are low in cost but high criticality. Since the carrying cost of these items (CV) is very low to decrease their ordering cost and to prevent their stock out they can be purchased once a year. Another subgroup of category I in this zone contains two items (Tramadol $50 \mathrm{mg}$ capsule and Paracetamol $500 \mathrm{mg}$ tablet) with $1.2 \%$ of TPE. These two items consumed a medium percentage of TPE but they were non-essentials. Since they are expensive and non-essential, AN class items should be monitored to save the budget and their placement of order should be done after careful evaluation of the items need. ${ }^{24,26,28}$ Rational use of items in this subgroup, including their removal from the list if possible and substitution with equal therapeutic medicine but less cost can bring about substantial saving without affecting patient care. ${ }^{24,28}$

The findings of this study were also comparable in terms of percentages of pharmaceutical expenditures with the study conducted at Saint Paul Hospital Millennium Medical College (Ethiopia) for 2013/14 to 2015/16 in which category I, II, and III items consumed around $85 \%, 12 \%$ and less than $1 \%$ of annual pharmaceutical expenditures in every three years. ${ }^{28}$ The similarity might be due to the occurrences of these health facilities in the same country which treat similar cases and might use similar pharmaceutical products.

The finding of this study had a gap as compared to the study conducted at tertiary care neuropsychiatry hospital in India where category I items $(33.8 \%)$ consumed $92.33 \%$ of pharmaceutical expenditures, category II items $(60 \%)$ and category III items $(6.2 \%)$ consumed $7.29 \%$ and $0.38 \%$ of annual pharmaceutical expenditures respectively. ${ }^{29}$ The present study had also a difference with another study conducted by Nigah et al ${ }^{24}$ in which $22.09 \%$ category I items consumed 
$74.21 \%$ of pharmaceutical expenditures, $54.63 \%$ category II items consumed $22.23 \%$ and $23.28 \%$ category III items consumed $3.56 \%$ of pharmaceutical expenditures. This variation might be due to the difference in the level of the health care settings, each health facility may use different pharmaceutical products and pharmaceuticals budget of each health facility.

\section{Conclusion}

This study assessed the pharmaceutical Inventory management facilities of the West Shewa zone by using ABC-VEN matrix analysis. The ABC analysis of selected health facilities of the West Shewa zone revealed that around $10 \%$ of pharmaceuticals consumed $70 \%$ of TPE and they need continuous day-to-day control. VEN analysis of pharmaceuticals showed that one-third of them were categorized as vital items and they consumed more than half of the TPE of this zone and they should not be stocked out. ABC-VEN matrix analysis of the study identifies category I items that consumed more than three- fourth of TPE and which need great attention for control while forecasting, selecting, purchasing, and keeping their stock. Therefore, Health facilities needs to conduct ABC-VEN matrix analysis of pharmaceuticals yearly to identify categories of pharmaceuticals requiring stringent control to enhance effective patient care and efficient use of resources.

\section{Abbreviations}

ABC, Always Better Control; DTC, Drug and Therapeutic Committee; ETB, Ethiopian Birr; HCMIS, Health Commodity Management information System; HC, Health Center; TPE, Total pharmaceuticals expenditures; VEN, Vital, Essential, Nonessential/Desirable.

\section{Data Sharing Statement}

The authors declare that the data supporting the finding of this study are available within article.

\section{Ethical Approval and Consent to Participate}

Ethical clearance was obtained from Ethical Review board of the Institute of Health Science, Jimma University (Ref.No: JURPGD/340/19). A permission letter was written for the West Shewa zone health department. The zonal health department then wrote permission letter to the district health offices and Hospitals. District Health Offices wrote a letter to HCs. A permission letter was obtained from HCs' administrative officers and taken to the pharmacy department and would inform other departments of the facility where data was collected. Before any data collection, the main purpose of the study was clearly explained to each department (concerned personnel). The written informed consent was obtained from the pharmacy professionals assigned to the pharmacy department of each health facility before the commencement of the study.

\section{Acknowledgment}

The authors would like to acknowledge Ambo University and Jimma University for sponsoring.

\section{Author Contributions}

All authors have made significant contribution to the work reported, that is conception, study design, execution, acquisition, of data, analysis and interpretation; have also participated in drafting or writing of the manuscript and critically revised the final version of the manuscript. All have agreed to the journal which the article has been submitted and agreed to be accountable for the content of the article.

\section{Funding}

The study was funded by Ambo University and Jimma University. The funding organization participated only in providing the material and financial support for the research. The funding body did not participate in the design of the study; data collection, analysis, interpretation and writing the manuscript. 


\section{Disclosure}

The authors declare that they have no competing interests.

\section{References}

1. Dwivedi S, Kothiyal P. Inventory management: a tool of identifying items that need greater attention for control. Pharm Innov. 2012;1(7):125.

2. Anand T, Ingle GK, Kishore J, Kumar R. ABC-VED analysis of a drug store in the Department of Community Medicine of a Medical College in Delhi. Indian J Pharm Sci. 2013;75(1):113-117. doi:10.4103/0250-474X.113543

3. Management Sciences for Health. MDS-3: Managing Access to Pharmaceuticals and Health Technologies. Arlington: Lynne Rienner Publishers; 2012.

4. Singam A, Dudhgaonkar S, Mamarde A, Salwe K, Khan H. ABC-VED analysis of drug store in tertiary care hospital for year 2013-14. Indo Am J Pharm Res. 2016;6(8):6-11.

5. Antonoglou D, Kastanioti C, Niakas D. ABC and VED analysis of medical materials of a general military hospital in Greece. $J$ Health Manag. 2017;19(1):170-179. doi:10.1177/0972063416682643

6. Sharma S. Tools for assessing and monitoring medicine use. In: Pharmaceutical Medicine and Translational Clinical Research. Elsevier; 2018:445-463.

7. Migbaru S, Yigeremu M, Woldegerima B, Shibeshi W. ABC-VEN matrix analysis of pharmaceutical inventory management in Tikur Anbessa Specialized Hospital for the years 2009 to 2013, Addis Ababa, Ethiopia. Indian J Basic Appl Med Res. 2016;5(2):734-743.

8. Pandya B, Thakkar H. A review on inventory management control techniques: ABC-XYZ analysis. REST J Emerging Trends Model Manuf. 2016;2:82-86.

9. Taddele BW, Wondimagegn AA, Asaro MA, Sorato MM, Gedayi BG, Hailesilase AA. ABC-VEN matrix analysis of the pharmacy store in a secondary level health care facility in Arbaminch Town, Southern Ethiopia. J Young Pharm. 2019;11(2):182. doi:10.5530/jyp.2019.11.38

10. Mohammed SA, Workneh BD. Critical analysis of pharmaceuticals inventory management using the ABC-VEN matrix in Dessie referral Hospital, Ethiopia. Integr Pharm Res Pract. 2020;9:113-125. doi:10.2147/IPRP.S265438

11. Nadu T. Drug inventory control analysis in a Primary level Health care facility in Rural Tamil Nadu, India. Age. 2012;5(2):36-40.

12. Devarajan D, Jayamohan M. Stock control in a chemical firm: combined FSN and XYZ analysis. Proc Technol. 2016;24:562-567. doi:10.1016/j. protcy.2016.05.111

13. Reddy DKVK, Sai DMSS, Prabhu DR. A study on the selective controls of inventory management and application of ABC XYZ Control Matrix in the Cardiology Department of a Tertiary Care Hospital. IOSR J Dent Med Sci. 2017;16(5):06-09. doi:10.9790/0853-1605090609

14. World Health Organization. Equitable Access to Essential Medicines: A Framework for Collective Action. World Health Organization; 2004.

15. Hogerzeil HV, Mirza Z. The World Medicines Situation 2011: Access to Essential Medicines as Part of the Right to Health. Vol. 680. Geneva: World Health Organization; 2011:689.

16. Droti B, O’Neill KP, Mathai M, Yao Tsidi Dovlo D, Robertson J. Poor availability of essential medicines for women and children threatens progress towards Sustainable Development Goal 3 in Africa. BMJ Glob Health. 2019;4(Suppl 9):e001306-e001306. doi:10.1136/bmjgh-2018-001306

17. Nditunze L, Makuza S, Amoroso CL, et al. Assessment of essential medicines stock-outs at health centers in Burera District in Northern Rwanda. Rwanda J. 2015;2(1):85-88. doi:10.4314/rjhs.v2i1.12F

18. Kefale AT, Shebo HH. Availability of essential medicines and pharmaceutical inventory management practice at health centers of Adama town, Ethiopia. BMC Health Serv Res. 2019;19(1):1-7. doi:10.1186/s12913-019-4087-0

19. Gebremariam ET, Gebregeorgise DT, Fenta TG. Factors contributing to medicines wastage in public health facilities of South West Shoa Zone, Oromia Regional State, Ethiopia: a qualitative study. J Pharm Policy Pract. 2019;12(1):1-7. doi:10.1186/s40545-019-0192-z

20. Central Statistical Authority. 2007 Population and Housing Census of Ethiopia Addis Ababa, Ethiopia; 2007.

21. Snow J. Logistics Indicators Assessment Tool (LIAT). Arlington, VA: John Snow, Inc./DELIVER, for the U.S. Agency for International Development; 2005.

22. Kant S, Haldar P, Singh A, Kankaria A. Inventory management of drugs at a secondary level hospital associated with Ballabgarh HDSS-An experience from North India. J Young Pharm. 2015;7(2):113-118. doi:10.5530/jyp.2015.2.9

23. Ceylan Z, Bulkan S. Drug inventory management of a pharmacy using ABC and VED analysis. Eurasian J Health Technol Assess. 2017;2(1):14-18.

24. Nigah R, Devnani M, Gupta A. ABC and VED analysis of the pharmacy store of a tertiary care teaching, research and referral healthcare institute of India. J Young Pharm. 2010;2(2):201-205. doi:10.4103/0975-1483.63170

25. GünerGören H, Dağdeviren Ö. An excel-based inventory control system based on ABC and VED analyses for pharmacy: a case study. Galore Int $J$ Health Sci Res. 2017;2(1):11-17.

26. Abate S. Assessment of Pharmaceuticals Inventory Management Systems for the Years $(2008,2009,2010)$ Using ABC-VEN Matrix Analysis at Addis Ababa University College of Health Sciences Tikur Anbessa (Black Lion) Specialized Hospital. Pharmacoepidemiol Drug Saf. 2012;2 (3): 1000137

27. Mousnad MA, Ibrahim MIM, Palaian S, Shafie AA. Medicine expenditures in Sudan National Health Insurance Fund: an ABC-VEN analysis of 5-year medicine consumption. J Pharm Health Serv Res. 2016;7(3):165-171. doi:10.1111/jphs.12136

28. Legese N. Pharmaceutical Expenditure Analysis and Assessment of Pharmaceutical Inventory Control Management Practices in Saint Paul Hospital Millennium Medical College. Addis Ababa, Ethiopia: School of Pharmacy, Addis Ababa University; 2017.

29. Khurana S, Chhillar N, Gautam VKS. Inventory control techniques in medical stores of a tertiary care neuropsychiatry hospital in Delhi. Health. 2013;5(01):8. doi:10.4236/health.2013.51002 


\section{Publish your work in this journal}

Integrated Pharmacy Research and Practice is an international, peer-reviewed, open access, online journal, publishing original research, reports, reviews and commentaries on all areas of academic and professional pharmacy practice. This journal aims to represent the academic output of pharmacists and pharmacy practice with particular focus on integrated care. All papers are carefully peer reviewed to ensure the highest standards as well as ensuring that we are informing and stimulating pharmaceutical professionals. The manuscript management system is completely online and includes a very quick and fair peer-review system, which is all easy to use. Visit http://www.dovepress.com/testimonials.php to read real quotes from published authors.

Submit your manuscript here: http://www.dovepress.com/integrated-pharmacy-research-and-practice-journal 